hausted by it; and Mr. H. N. Dickson his charts and sections showing the temperature of the water in the northern and western parts of the North Sea and the Faroe-Shetland Channel at all depths, August 1893 .

Dr. H. R. Mill and Mir. Edward Heawood exhibited bathymetrical maps of Windermere, Ullswater, Coniston Vater, Derwentwater and Bassenthwaite, Buttermere and Crummock, Ennerdale Water, Wastwater and Haweswater. Contour lines at each $25^{\text {sfeet }}$ of depth beneath the surface were shown, and the configuration of the basins was thus for the first time accurately delineated.

Prof. J. Norman Lockyer exhibited photographs of stellar spectra taken with a 6 -inch objective prism of $45^{\circ}$, and photographs of the great sun-spot of February 1894, taken at Dehra Dun.

Living larvæ infuenced by the colours of their surroundings were exhibited by Prof. E. B. Poulton; and microscopic slides illustrating the behaviour of the nucleus during spore formation in the hepatice, by Prof. I. B. Farmer.

Photographs of diffraction and allied phenomena were exhibited by MIr. WV. B. Croft. The photographs showed Newton's rings, reflected and transmitted; Grimaldi's fringes; Fresnel's interference from a bi-prism; Arago's shifting of bands towards the denser medium; Talbot's bands. The shadows of needles, wire gauze, perforated zinc, a screen with circular holes, opaque circular screens with Arago's bright centre. A comparison of the diffraction of Fresnel with that of Fraunhofer and Schwerd; the diffracting ob:ect consists of groups of small circles of light. Uniaxal and biaxal crystals; conical refraction.

Specimens demonstrating some phenomena of chemiotaxis in inflammation were exhibited by Mr. IV. B. Hardy and Dr. A. A. Kanthack.

Prof. Marshall Ward showed apparatus employed for observing and measuring the growth of bacteria, fungi, and other micro-organisms under different conditions under the microscope. The essential feature is the culture-cell. It has a quartz floor, and is capable of holding large quantities of water, and thus while letting the light-rays pass does not rapidly vary in temperature. By the side of the culture-cell containing the hanging-drop in which is the organism under observation, is an exactly similar cell, but with a small thermometer in it, the blackened bulb of which is in the cell, and gives the temperature inside the latter. The rest of the apparatus consists in the measuring eye-piece; the screens of coloured glass, various liquias, \&c., for growth in different kinds of light ; and a warm chamber in which the whole microscope can be enclosed and kept at known temperature.

A demonstration of the trails of Oscillatorix formed the exhibit of Mr. J. G. Grenfell.

Prof. E. Waymouth Reid exhibited microscopic specimens illustrative of the process of secretion in the skin of the eel. The chief point of general interest in the process is the peculiar manner in which the surface of the skin is cast off when the animal is stimulated.

Prof. G. B. Howes exhibited eggs and young of Ceratodus For steri, and a male of Lepidosircu paradoxa.

Mr. E. J. Allen showed nerve elements from the ganglia of lobster embryos; and Dr. D. Sharp a collection of white ants Termitide).

A specimen and drawing of the South American mud-fish, Lepidosircn paradoxa, was exhibited by Prof. E. Ray Lankester. (See NATURE, vol. xlix. p. 555.)

Dr. Alexander Muirhead exhibited a new form of Lord Kelvin's siphon recorder, Mruirhead's artificial cable, and Muir. head's automatic curb transmitter. Lord Kelvin's siphon recorder and Muirhead's automatic curb transmitter were shown in operation in connection with an artificial cable of the same capacity and conductor resistance as the Atlantic cable, which is to be laid next July by the Anglo-American Telegraph Com. pany. Capacity of artificial cable 800 microfarads; resistance of conductor $335 \circ$ B.A. units.

Demonstrations by means of the electric lantern took place during the evening, Dr. D. H. Scott showing photographs from sections in Dr. WV. C. WVilliamson's collection, illustrat. ing the microscopic structure of fossil plants from the coalmeasures. The lantern was also used by Prof. E. B. Poulton, who exhibited illustrations of recent work upon the influence of environment upon the colours of certain Lepidopterous larvæ. Variously coloured twigs and shoots, NO. I 280 , voL. 50 ] such as occur in nature, have been shown to influence the appearance of many twig-like larva in such a manner as to conceal them. During the summer of 1893 certain larvæ of two species (Gastrofacha quercifolta and Otontoptera bidentata) were surrounded, during their growth, with lichen-covered twigs. Larvæ thus treated developed lichen-like marks upon the body.

\section{THE RELATIVE SENSITIVITY OF MEN AND WOMEN AT THE NAPE OF THE NECK (BY WEBER'S TEST).}

THE difference in the sensitivity of the two sexes has been discussed often and from various points of view, but still, as it would seem, upon insufficient data. More observations being wanted, I submit the following, partly for such value as they have in themselves, partly to show an easy method of observation which others may pursue with advan. tage, and partly as a good illustration of the method of percentiles, or centiles.

The test employed is one of a familiar kind, made with the points of a pair of compasses, and usually associated with the name of Weber. If one person becomes just conscious of the doubleness of the pricks when the distance between the points is $a$, and another person does so when the interval is $b$, then the ratio of $a$ to $b$ may fairly be taken to express the relative obtuseness of the two persons, so far as concerns the form of sensitivity tested, and the inverse ratio of $b$ to $a$ to represent its relative delicacy. The particular test used was one that has three especial merits : it requires no minuteness of measurement, no uncovering, and the person tested is unable to see the operation. It consists in pressing the points of the compasses against the nape of the neck and across the line of the spine, while the experimentee sits with his or her head bowed forward. The just-perceptible interval at the nape of the neck averages as much as half an inch or thereabouts, while its variation in different persons is large. Consequently there is no need for extreme delicacy of measurement, neither does the varying thickness of cuticle caused by various degrees of usage, interfere materially with the results, as it does when like experiments are made, as is usual, on the finger-tips. The varying delicacy of perception due to differing amounts of practice is here entirely eliminated, because all persons are equally unpractised, no one occupying hiwself or herself in attempts to discriminate between two simultaneous pressures on the nape of his or her neck, while everybody has life-long practice in discriminating roughnesses, though in various and unascertainable degrees, with his or her finger-tips. There are parts of the body, such as the back, which are still less discriminative than the nape of the neck, but there is no other equally suitable part that is so get-at-able, in respect to the the ordinary dress of man or woman. Lastly, the attitude of the person who is being tested, entirely precludes him from watching the operator, and guessing from the hands or movements of the latter, whether he is applying two points, or only one, at the moment when he asks what is felt. The observations were all made by Sergeant Randall, who superintends my laboratory; he employed the two points of a Flower's craniometer, which was handy for use, as it was wanted to make other measurements of the same persons. The observations were carried on for some months, until a sufficient number had accumulated to justify discussion. Stature was included among them, but, failing on examination to trace any notable relation between stature and the just-perceptible interval on the nape of the neck, I have disregarded stature altogether in the following summary, and age too, so far that the person tested was often not fully grown.

The observations made on males and females, respectively, are summarised in the first and third lines of Table I. Their sums, reckoned in each case from the beginning of the series, are entered in lines 2 and 4 , while the percentages of those sums are given in lines 3 and 6 , but solely for the purpose of graphic projection in the form of dots, in Fig. 1. Those dots are joined by straight lines, forming traces for the males and females respectively. The lengths of the ordinates to the traces, which are drawn at the 10th, 20th, \&c. divisions of the base, are the 1oth, 2oth, \&c. percentiles, or centiles; or, in still briefer language, the Ist, 2nd, \&c. "deciles." Their values, obtained by simple interpolation from the entries in lines 
I and 2 of Table I., are entered in Fig. I. Thus 13.8 millimetres is the just-perceptible interval of the median man, and II. 8 that of the median female. In one sense, but only in an imperfect one, the relative sensitivity of the two sexes is given by these figures as being about 7 to 6 . Much more has, how- observed values could be obtained by closer attention to the second decimals, but such minuteness is uncalled for in a case like this. It will be seen from columns $\mathrm{B}, \mathrm{C}$, and $b, c$, in Table II., that the sums of the observed and calculated deciles closely accord, and that the differences between the several

TABLE I.

Summary of Observalions of 932 Males and 377 Females, showing the number in whom a just-perceftible feeling of doubleness was given by the pressure of two points across the nape of the neck, and separated by the various intervals, as below.

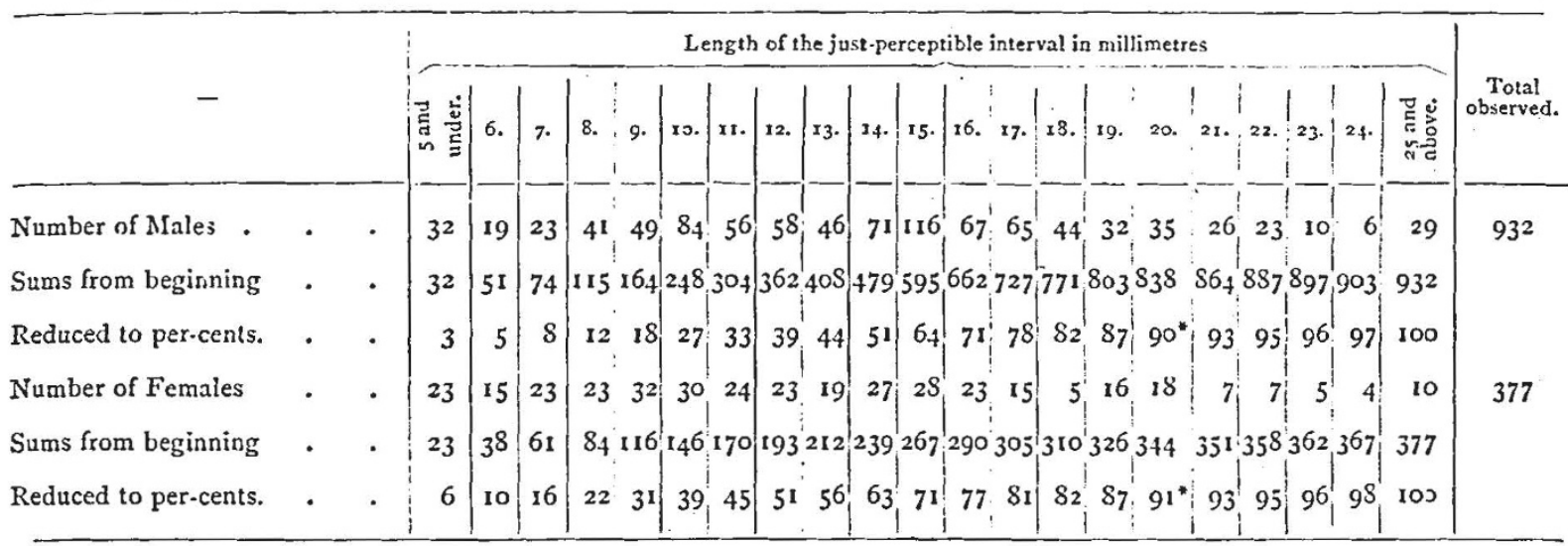

These figures are protracted in both cases as $90^{\circ} 5$, inasmuch as the accordance of the two preceding and of the:four subsequent entries make the correction reasonable as well as convenient.

ever, to be specified before the relation can be adequately expressed, because it is obvious from the diagram, that what is true for persons having medium sensitivity, is not true for those having high, and still less for those having low, sensitivity. We are, how-

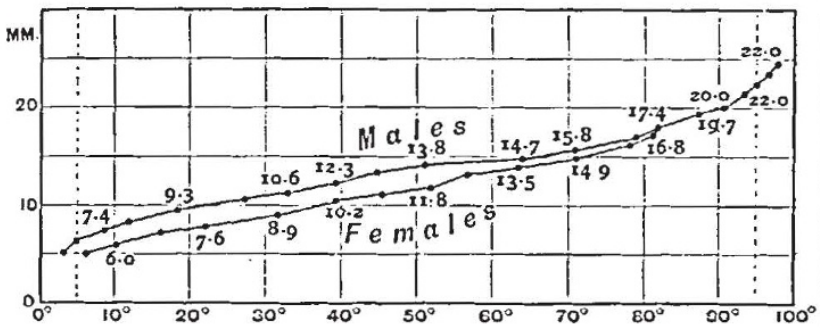

Fic. I. -Traces and deciles from observations. The dots refer to the observed values as given in the 3 rd and 6 th lines of the table. They are connected by straight lines. The figures are the values of the corresponding deciles - that is, of the ordinates to the traces erected at each successive tenth part of the base.

ever, able to specify what is wanted very compendiously, because both of the traces conform fairly well to the law of frequency of error, at least between the limits of the Ist and the gth decile. In the case of mal s, the median is taken at 3.50 millimetres, and

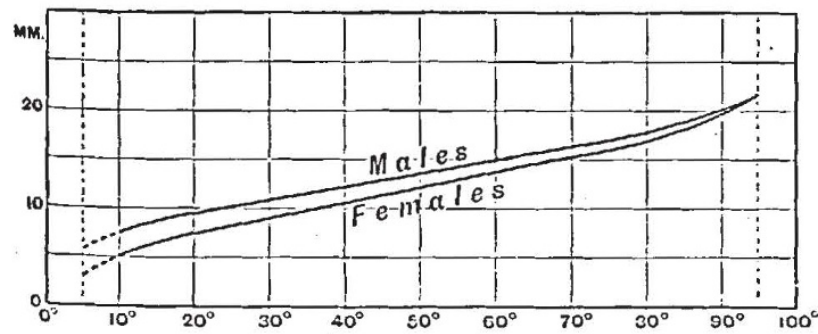

Fic. 2.-Deciles and curves by calculation.

Data for males : median $=13^{\circ} 50, q=3.25 \mathrm{~mm}$

Data for females : median $=12^{\circ} \infty, q=3^{\circ} 7^{\circ} \mathrm{mm}$.

the $q$ (= half the difference between the two quartiles, a value which is identical with that of the \pm probable error of a single observation) at 3.25 ; in females, the corresponding values are 12.00 and 370 . A somewhat nearer approximation to the No. I 280 , vor. 50$]$ pairs of them, headed $\mathrm{B}-\mathrm{C}$ and $b-c$, are as nearly alike as we have a right to expect. The calculated deciles, and the curves drawn through them, in Fig. 2, may therefore be accepted as a just rendering of what is more roughly indicated by the observations in Table I. and Fig. I. In the following remarks reference will be made almost exclusively to the calculated values, but the results can and will usually be checked by reference to the observed ones, with which they tally sufficiently well.

TABLE II.

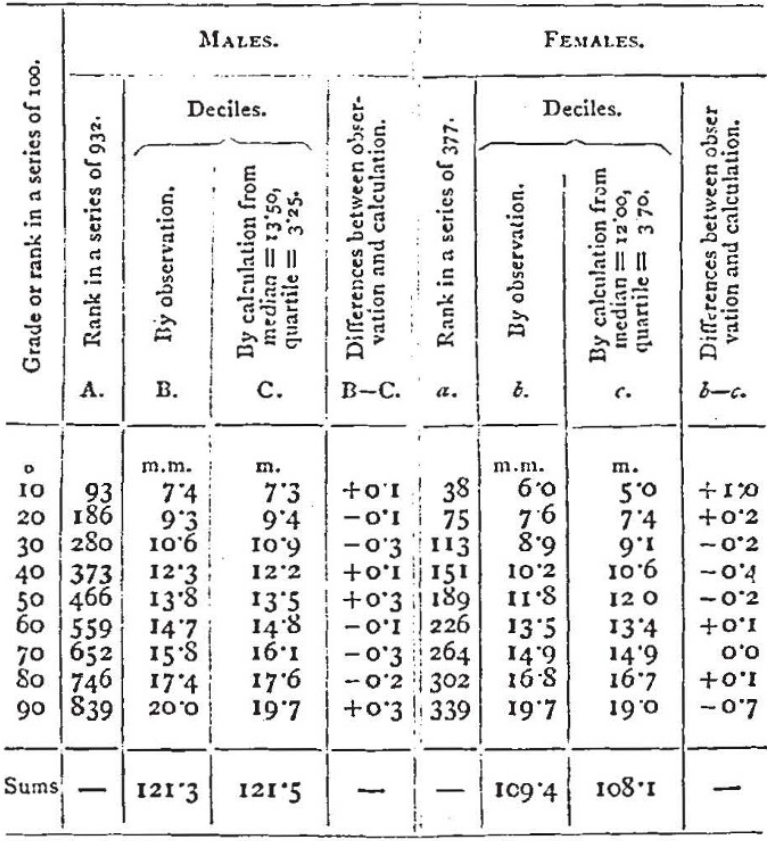

The average ratio between the sensitivities of the females and males is the same as that between the sums (or means) of columns $\mathrm{C}$ and $c$ in Table II., namely as 125.5 to 108.1 ; or to speak more modestly, as no trust can be reposed on the minute pre- 
cision of such values as these, the average delicacy of female discrimination between the two points is to that of the male, in a ratio that lies somewhere between 7 to 6 and 8 to 7 , or thereabouts. It will be recollected that the former ratio was that between the median female and the median male.

The variability of the discriminative power appears from the observations to be distinctly higher among females than among males. Measuring it in the usual way, by the half difference between the two quartiles, which is the same thing as the froballe error of a single observation, or else by any multiple of this, as by the mean error, we find that the variability among females is to that among males as $3 \cdot 70$ to $3 \cdot 25$, say as 8 to 7 . It is in consequence of this that so large a difference is shown between the relative sensitivity of the two sexes, at the right and left extremes of their respective curves in Fig. 2. We find from Table II. that the value of $\mathrm{C}-c$ at the Ist decile is 2.3 millimetres, and at the 9 th decile it is only $0 \% 7$, the differences between the intermediate pairs decreasing regularly. The regularity of the decrease is not apparent in the actual obser. vations, as shown in Fig. I, nor in Table I., still there is nothing in what we see there that is incompatible with Fig. 2, while the fact of the difference between the right ends of the traces being much less than that between the left, is conspicuous.

Is it, however, a physiological fact that women are more variable than males in respect to discriminative touch, or are the observations affected by any exfraneous cause of variability? I think that the recorded variability may in a very small part be accounted for by the fact that women vary much more than men in the exercise of sustained attention. Carelessness would affect the results in the same direction as diminished sensitivity. Thus suppose one part of a large number of persons who were all really alike in sensitivity, to be very careless, and the remainder to be scrupulously careful; the minds of the careless would be apt to wander; they would then fail to notice the first just-perceptible sense of doubleness, and would appear, in consequence, to be more obtuse than the careful ones. Though the range of variability was in reality nil, the existence of carelessness would introduce variability into the records. Some women are religiously painstaking, as much so as any men; but the frivolity of numerous girls, and their incapacity of, or un. willingness to give, serious attention, is certainly more marked than among men of similar ages. Women may, however, be really more variable than men in respect to sensitivity, because they seem more variable in a few other respects, such as in stature and obesity. Many more very tall girls are to be seen now-adays among the upper classes than formerly, but the run of the statures among men has not altered quite so much. The multitude of extraordinarily obese women who used to frequent Vichy for the cure of fatness, were wonderful to behold ; but they are no longer to be seen in their former abundance, as the fashion of treatment has changed within recent years. Again, it appears that women vary much more widely than men in respect of their morality; to which assertion I would quote Tennyson as a corroborative witness, who writes as follows, in Merlin's soliloquy on the character of Vivien :-

$$
\begin{aligned}
& \text { "For men at most differ as heaven and earth, } \\
& \text { But women best and worst as heaven asd hell." }
\end{aligned}
$$

Since Fig 2 is true to scale, it is easy to utilise it for ascertaining the class.place of any man or woman in respect to the form of sensitivity now in question. The whole process would be as follows:-Take a pair of compasses, and find with them by experiment the just-perceptible interval across on the nape of the neck of the person tested then apply the compasses, to Fig. 2, keeping one (the lower) of its points always on the base line of the Fig., and holding the compasses so that the line joining its points shall be perpendicular to that base line. Slide the lower point of the compasses along the base line until the upper point touches the male or female trace, as the case may be; then read off the grade at which the lower point stands on the base line. Suppose it to be $35^{\circ}$; we thereby learn that 35 per cent. of the same sex have more sensitivity than the person tested, and that 65 per cent. have less. Similarly for any other value.

It would, I think, be well worth the while of an inquirer to repeat these tests, to revise my results, and to pursue the subject much further. If any one should feel disposed to do so, I would suggest that he should make his measurements with the cheap form of bow compasses, in common use by carpenters. The legs are connected not by a joint, but by a spring which tends to separate them, and they are brought together to any desired No. 1280 , voL. 50$\rceil$ interval hy turning a screw with the finger and thumb, which overcomes the spring. The interval between the points could eavily be measured on a separate scale; all the more easily, if there were a slight depression at the zero point of the scale, in which one leg wight be securely rested.

Francis Galton.

\section{THE RELATION OF MATHEMATICS TO ENGINEERING. ${ }^{1}$}

M ATHEMATICS has been described in this room as a good servant but a bad master. It will be my duty this even. ing to prove by suitable illustration the first half of the proposition, and to show the service mathematics has rendered and can render to engineers and engineering.

In our charter the Institution of Civil Engineers is defined as "A society for the general advancement of Mechanical Science, and more particularly for promoting the acquisition of that species of knowledge which constitutes the profession of a Civil Engineer, being the art of directing the great sources of power in nature for the use and convenience of man, as the means of production and of traftic in states both for external and internal trade, as applied in the construction of roads, bridges, aqueducts, canals, river naviyation and docks, for internal intercourse and exchange, and in the construction of ports, harbours, moles, breakwaters and lighthouses, and in the art of navigation by artificial power for the purposes of commerce, and in the construction and adaptation of machinery, and in the drainage of cities and towns." No better definition can, I think, be found for our profession than that it is the art of directing the great sources of power in nature for the use and convenience of man. It covers all that the widest view of our work can include, and it excludes those applied sciences, such as medicine, which deal with organised beings. Mathematics has to deal with all questions into which measurement of relative magnitude enters, with all questions of position in space, and of accurate deter. mination of shape. Engineering is a mathematical science in a peculiar sense. Medicine, the other great profession of applied science, has but little to do with questions of measurement of magnitudes, or of geometry; but the engineer finds them enter into everything with which he has to deal, and enter in the most diverse ways. The thing he has to determine is that the means he employs is enough and not unnecessarily more thar enough to attain the end in view. For this he must numerically measure the end and the means and see that they are justly proportioned to each other. It is useless this evening to waste time proving, what all will admit, that nu one can be even the humblest engineer without a knowledge of arithmetic and enough of geometry to enable him to read a drawing, that some trigonometry, some rational mechanics and a knowledge of projections, is a very useful part of the mental equipment of a draughtsman. It is hardly necessary to call attention to the great economy in the labour of calculations effected by the use of logarithms, a mathematical instrument for which we are indebted to Napier. We may with more profit examine what u-e the higher mathematics can be to the practical engineer, and what has been done in the past for engineering by its aid.

Judging from etymology, mathematics must have been begun by engineers; for surely geometry is the work of the earth measurer or land surveyor. But since the prehistoric times when geometry was initiated, engineers have not added much that is new to mathematics. They have rather sought amidst the stores of the mathematician and selected the handiest mathematical tool they could find for the particular purpose of the moment, but have done little or nothing in return in the way of improving the tools which they borrow. In this respect the relation of engineering to mathematics differs much from its relation to experimental physics. In electricity, magnetism and heat, engineers have from their practical experience repeatedly corrected the icleas of the theorists, and have started the science on more accurate lines. If our subject to-night had been the use of the practical applied science of engineering in promoting the development of pure mathematics, we should speedily find that there was hardly any materiai for discussion. The account being all on one side, let us see to what the debt of the engineer to the mathematician amounts.

There is no department of practical engineering in which the

1 The "James Forrest" Lecture, delivered at the Institution of Civil Engineers, by Dr. John Hopkinsin, F.R.S., on Mlay 3. 